\title{
Save the data! Diffuse scattering to shed light on structural dynamics
}

\author{
Michael E. Walla \\ ${ }^{a}$ Computer, Computational, and Statistical Sciences Division, Los Alamos National Laboratory, \\ Los Alamos, New Mexico 87545, USA mewall@lanl.gov
}

Structural dynamics in crystalline proteins gives rise to diffuse scattering - intensity beneath and away from the Bragg peaks in diffraction images. Recent improvements in X-ray beamlines and detectors are creating unprecedented opportunities for using macromolecular diffuse scattering to understand crystalline protein dynamics; however, modern data collection methods are hiding the data from users. This talk will examine challenges in processing, archiving, and modeling diffuse data obtained at modern light sources. For example, a major challenge is to enable users to see whether they have obtained high-quality data in real time, enabling useful adjustments to be made during the crystallographic experiment. Such feedback is also important during experiments focused purely on Bragg analysis, so that raw data with interesting diffuse features can be identified and preserved without corruption for future studies. 